\title{
Correction to: Synchronization of coupled neural networks with infinite-time distributed delays via quantized intermittent pinning control
}

\author{
Xinsong Yang@ - Yuming Feng - Ka Fai Cedric Yiu - Qiang Song • \\ Fuad E. Alsaadi
}

Published online: 30 July 2018

(C) Springer Nature B.V. 2018

\section{Correction to: Nonlinear Dyn}

$$
\text { https://doi.org/10.1007/s11071-018-4449-x }
$$

The third author's name was incomplete and incorrect in the original publication. It is correctly shown here.

The original article has been corrected.

The original article can be found online at https://doi.org/10.

1007/s11071-018-4449-x.

\footnotetext{
X. Yang $(\varangle)$

School of Mathematical Sciences, Chongqing Normal

University, Chongqing 401331, China

e-mail: xinsongyang@163.com

Y. Feng

Key Laboratory of Intelligent Information Processing and

Control of Chongqing Municipal Institutions of Higher

Education, Chongqing 404100, China

e-mail: yumingfeng25928@163.com

K. F. C. Yiu

Department of Applied Mathematics, Hong Kong

Polytechnic University, Hong Kong, China

e-mail: macyiu@polyu.edu.hk

Q. Song

College of Electrical Engineering, Henan University of

Technology, Zhengzhou 450001, China

e-mail: songqhdu@163.com

F. E. Alsaadi

Faculty of Engineering, King Abdulaziz University, Jeddah

21589, Saudi Arabia

e-mail: fuad-alsaadi@yahoo.com
} 